\title{
Structural Phase Transitions and Metallized Phenomena in Arsenic Telluride under High Pressure
}

Jinggeng Zhao, ${ }^{*}{ }^{\dagger}$ Liuxiang Yang, ${ }^{\S} \|$ Zhenhai Yu, ${ }^{\S}$ Yong Wang, ${ }^{\ddagger}$ Chunyu Li, ${ }^{\S}$ Ke Yang, ${ }^{\perp}$ Zhiguo Liu, ${ }^{*} *$ and Yi Wang $*, \dagger$

${ }^{\dagger}$ Natural Science Research Center, Academy of Fundamental and Interdisciplinary Sciences, and Department of Physics, Harbin Institute of Technology, Harbin 150080, China ${ }^{\S}$ Center for High Pressure Science and Technology Advanced Research, Shanghai, 201203, China

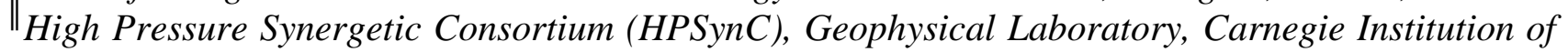
Washington, Argonne, Illinois 60439, United States

${ }^{\perp}$ Shanghai Institute of Applied Physics, Chinese Academy of Sciences, Shanghai 201203, China

E-mail: zhaojinggeng@163.com (J.G.Z.), liuzhiguo@hit.edu.cn (Z.G.L.), yw@ @it.edu.cn (Y.W.). 
Table S1. Experimental lattice parameters and unit cell volumes of $\mathrm{As}_{2} \mathrm{Te}_{3}$ under high pressure

\begin{tabular}{|c|c|c|c|c|c|c|}
\hline Phase & $P(\mathbf{G P a})$ & $a(\AA)$ & $b(\AA)$ & $c(\AA)$ & $\beta\left({ }^{\circ}\right)$ & $V\left(\AA^{3}\right)$ \\
\hline$\alpha$ & 0 & $14.3884(14)$ & $4.0318(2)$ & $9.9233(7)$ & $94.96(1)$ & $573.51(7)$ \\
\hline$\alpha$ & 0.58 & $14.2856(13)$ & $4.0168(2)$ & $9.8490(8)$ & $95.11(1)$ & $562.92(5)$ \\
\hline$\alpha$ & 1.10 & $14.1793(15)$ & $4.0056(2)$ & $9.7962(9)$ & $95.22(1)$ & $554.09(7)$ \\
\hline$\alpha$ & 2.10 & $14.0453(19)$ & $3.9806(3)$ & $9.6861(12)$ & $95.47(1)$ & $539.07(6)$ \\
\hline$\alpha$ & 3.09 & 13.9231(13) & $3.9604(3)$ & $9.6085(9)$ & $95.58(1)$ & $527.31(5)$ \\
\hline$\alpha$ & 4.15 & $13.8243(15)$ & $3.9439(3)$ & $9.5378(12)$ & $95.50(1)$ & $517.63(7)$ \\
\hline$\alpha^{\prime}$ & 5.09 & $13.7653(13)$ & $3.9297(3)$ & $9.4835(12)$ & $95.61(1)$ & $510.28(5)$ \\
\hline$\alpha^{\prime}$ & 6.16 & $13.6969(13)$ & $3.9179(3)$ & $9.441(1)$ & $95.51(1)$ & $504.30(5)$ \\
\hline$\alpha^{\prime}$ & 7.16 & $13.6441(12)$ & $3.9041(3)$ & $9.3914(9)$ & $95.48(1)$ & $497.98(4)$ \\
\hline$\alpha^{\prime}$ & 8.17 & $13.6069(13)$ & $3.8934(3)$ & $9.3250(8)$ & $95.53(1)$ & $491.72(5)$ \\
\hline$\alpha^{\prime}$ & 9.18 & $13.5605(13)$ & $3.8810(3)$ & $9.2697(8)$ & $95.51(1)$ & $485.60(5)$ \\
\hline$\alpha^{\prime}$ & 10.3 & $13.5167(11)$ & $3.8713(4)$ & $9.2214(8)$ & $95.61(1)$ & $480.23(5)$ \\
\hline$\alpha^{\prime}$ & 11.3 & $13.4813(13)$ & $3.8559(4)$ & $9.1886(13)$ & $95.52(1)$ & $475.43(6)$ \\
\hline$\alpha^{\prime}$ & 12.2 & $13.4495(17)$ & $3.8459(4)$ & $9.1515(14)$ & $95.66(1)$ & $471.06(9)$ \\
\hline$\alpha^{\prime \prime}$ & 13.2 & $13.455(2)$ & $3.8401(7)$ & $9.071(2)$ & $95.50(1)$ & $466.52(12)$ \\
\hline$\alpha^{\prime \prime}$ & 14.6 & $13.462(2)$ & $3.8226(6)$ & $9.0004(14)$ & $95.34(1)$ & $461.17(12)$ \\
\hline$\alpha^{\prime \prime}$ & 15.5 & $13.482(3)$ & $3.8192(7)$ & $8.9320(16)$ & $95.21(2)$ & $458.03(13)$ \\
\hline$\alpha^{\prime \prime}$ & 16.3 & $13.519(2)$ & $3.8110(8)$ & $8.8674(14)$ & $95.02(2)$ & $455.10(11)$ \\
\hline$\alpha^{\prime \prime}$ & 17.3 & $13.539(2)$ & $3.8022(7)$ & $8.7980(15)$ & $94.95(2)$ & $451.23(8)$ \\
\hline$\alpha^{\prime \prime}$ & 18.4 & $13.574(4)$ & $3.7918(10)$ & $8.729(2)$ & $94.69(3)$ & $447.76(12)$ \\
\hline$\gamma$ & 16.3 & $9.513(3)$ & $6.9182(18)$ & $9.678(4)$ & $135.97(2)$ & $442.81(18)$ \\
\hline$\gamma$ & 17.3 & $9.482(3)$ & $6.8865(14)$ & $9.660(3)$ & $135.94(2)$ & $438.78(16)$ \\
\hline$\gamma$ & 18.4 & $9.469(2)$ & $6.8421(13)$ & $9.652(3)$ & $135.88(1)$ & $435.45(15)$ \\
\hline$\gamma$ & 19.4 & $9.445(2)$ & $6.8255(11)$ & $9.643(2)$ & 135.91(1) & $432.61(12)$ \\
\hline$\gamma$ & 20.3 & $9.427(3)$ & $6.7668(17)$ & $9.624(3)$ & $135.84(1)$ & $427.74(14)$ \\
\hline$\gamma$ & 21.5 & $9.408(4)$ & $6.7282(15)$ & $9.610(3)$ & $135.91(2)$ & $423.31(13)$ \\
\hline$\gamma$ & 23.0 & $9.398(4)$ & $6.6857(14)$ & $9.599(3)$ & $135.87(1)$ & $420.05(12)$ \\
\hline$\gamma$ & 24.6 & $9.359(3)$ & $6.6388(13)$ & $9.580(4)$ & $135.80(1)$ & $415.05(15)$ \\
\hline$\gamma$ & 26.5 & $9.326(3)$ & $6.5966(12)$ & $9.561(2)$ & $135.75(1)$ & $410.49(6)$ \\
\hline$\gamma$ & 29.0 & $9.298(2)$ & $6.5291(10)$ & $9.515(3)$ & $135.66(1)$ & 403.81(9) \\
\hline$\gamma$ & 30.6 & $9.252(3)$ & $6.4825(11)$ & $9.506(2)$ & $135.69(1)$ & $398.25(4)$ \\
\hline$\gamma$ & 33.4 & $9.225(2)$ & $6.4127(10)$ & $9.481(2)$ & $135.68(1)$ & 391.93(4) \\
\hline$\gamma$ & 36.6 & $9.170(3)$ & $6.3720(14)$ & $9.4377(16)$ & $135.68(1)$ & $385.38(4)$ \\
\hline$\gamma$ & 40.8 & $9.144(3)$ & $6.2898(11)$ & $9.4157(15)$ & $135.72(1)$ & $378.13(4)$ \\
\hline
\end{tabular}




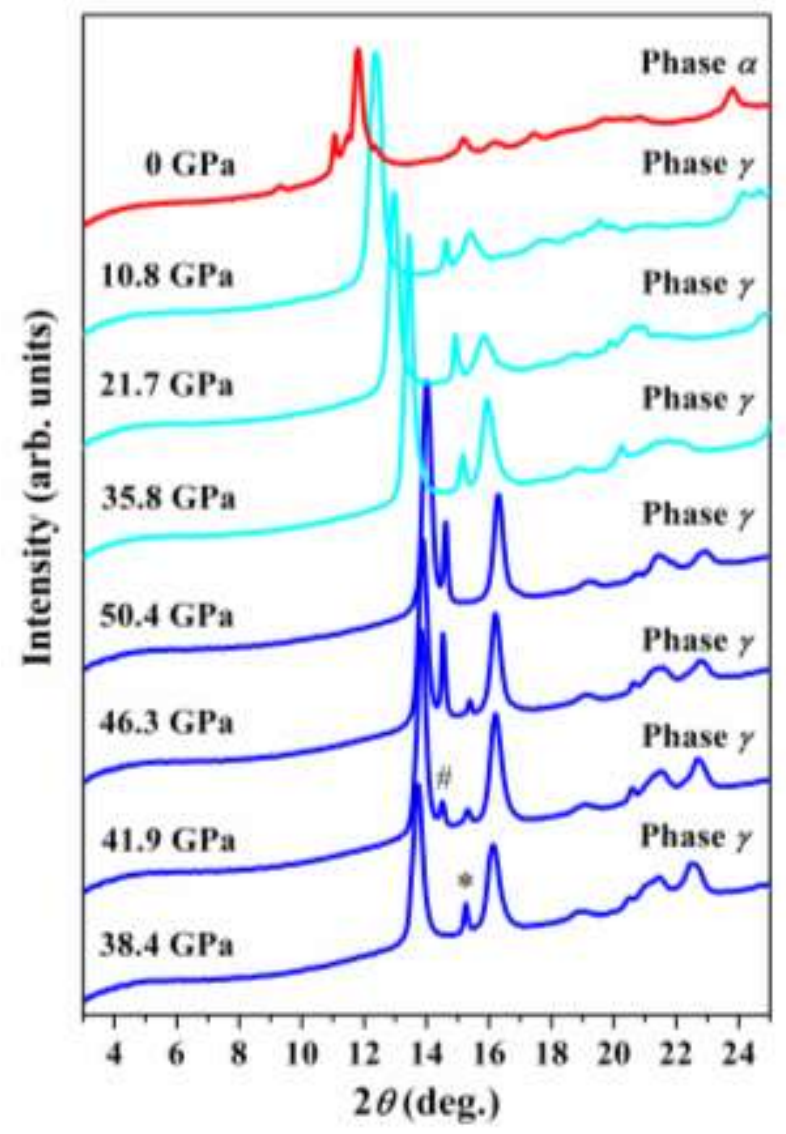

Figure S1. Selected angle-dispersive X-ray diffraction (AD-XRD) patterns of $\mathrm{As}_{2} \mathrm{Te}_{3}$ at room temperature in a loading process (blue lines) up to $50.4 \mathrm{GPa}$ and a unloading process (cyan and red lines) down to $0 \mathrm{GPa}(\lambda=0.6199 \AA)$.

\section{Notes:}

In Figure S1, with increasing pressure, the asterisk (*) peak weakens and disappears at $50.4 \mathrm{GPa}$, while the intensity of number sign (\#) peak increases upon compression. Due to the different width of these two peaks from those for phase $\gamma$, they do not belong to the high-pressure phases of $\mathrm{As}_{2} \mathrm{Te}_{3}$. At ambient pressure, there is not any impurity in the XRD pattern of the sample used in this work. In the unloading process, the number sign peak vanishes at $35.8 \mathrm{GPa}$ and the asterisk peak reemerges and keeps down to at least $10.8 \mathrm{GPa}$. As shown in Figure $\mathrm{S} 1, \mathrm{As}_{2} \mathrm{Te}_{3}$ recovers to the original structure at pressure release, which shows that the phase transition could be reversible.

For the binary compounds composed of the $V$ and $V I$ group elements ( $\mathrm{As}-\mathrm{Te}, \mathrm{Bi}-\mathrm{Te}, \mathrm{Sb}-\mathrm{Te}$, and $\mathrm{Bi}-\mathrm{Se}$ materials), the chemical composition and crystal structures are very complex. ${ }^{\mathrm{S} 1-\mathrm{S} 4}$ According to Ref. S1a, it is easy for $\mathrm{As}_{2} \mathrm{Te}_{3}$ to decompose other compounds under high-pressure and high-temperature conditions. Therefore, part of $\mathrm{As}_{2} \mathrm{Te}_{3}$ may decompose into a new compound 
indicated by the asterisk peak at high pressures and then this new material transforms into another phase (corresponding to the number sign peak) upon further compression. Because of lack of diffraction peaks and weak intensity of these new phases, it is difficult to obtain their chemical composition and crystal structures. Further high-pressure experiments would be performed at higher pressures to reveal the novel structural transitions in $\mathrm{As}_{2} \mathrm{Te}_{3}$.

\section{REFERENCES}

(S1) (a) Kirkinskii, V. A.; Yakushev, V. G. Inorg. Mater. 1974, 10, 1230-1233. (b) Yakushev, V. G.; Kirkinskii, V. A. Dokl. Akad. Nauk SSSR 1969, 186, 882-884. (c) Eifert, J. R.; Peretti, E. A.; J. Mater. Sci. 1968, 3, 293-296. (d) Quinn, R. K. Mater. Res. Bull. 1974, 9, 803-814.

(S2) (a) Nakajima, S. J. Phys. Chem. Solids 1963, 24, 479-485. (b) Yamana, K.; Kihara, K.; Matsumoto, T. Acta Crystallogr. 1979, B35, 147-149. (c) Feutelais, Y.; Legendre, B.; Rodier, N.; Agafonov, V. Mater. Res. Bull. 1993, 28, 591-596.

(S3) (a) Anderson, T. L.; Krause, H. B. Acta Crystallogr. 1974, B30, 1307-1310. (b) Kifune, K.; Kubota, Y.; Matsunaga, T.; Yamada, N. Acta Crystallogr. 2005, B61, 492-497. (c) Ullner, H. A. Ann. Phys. 1968, 21, 45-56. (d) Agafonov, V.; Rodier, N.; Ceolin, R.; Bellissent, R.; Bergman, C.; Gaspard, J. P. Acta Crystallogr. 1991, C47, 1141-1143.

(S4) (a) Zhou, W. Y.; Meetsma, A.; De Boer, J. L.; Wiegers, G. A. Mater. Res. Bull. 1992, 27, 563-572. (b) Glazov, V. M. Inorg. Mater. 1984, 20, 1068-1074. (c) Gaudin, E.; Jobic, S.; Evain, M.; Brec, R.; Rouxel, J. Mater. Res. Bull. 1995, 30, 549-561. (d) Okamoto, H. J. Phase Equilib. 1994, $15,195-201$. 


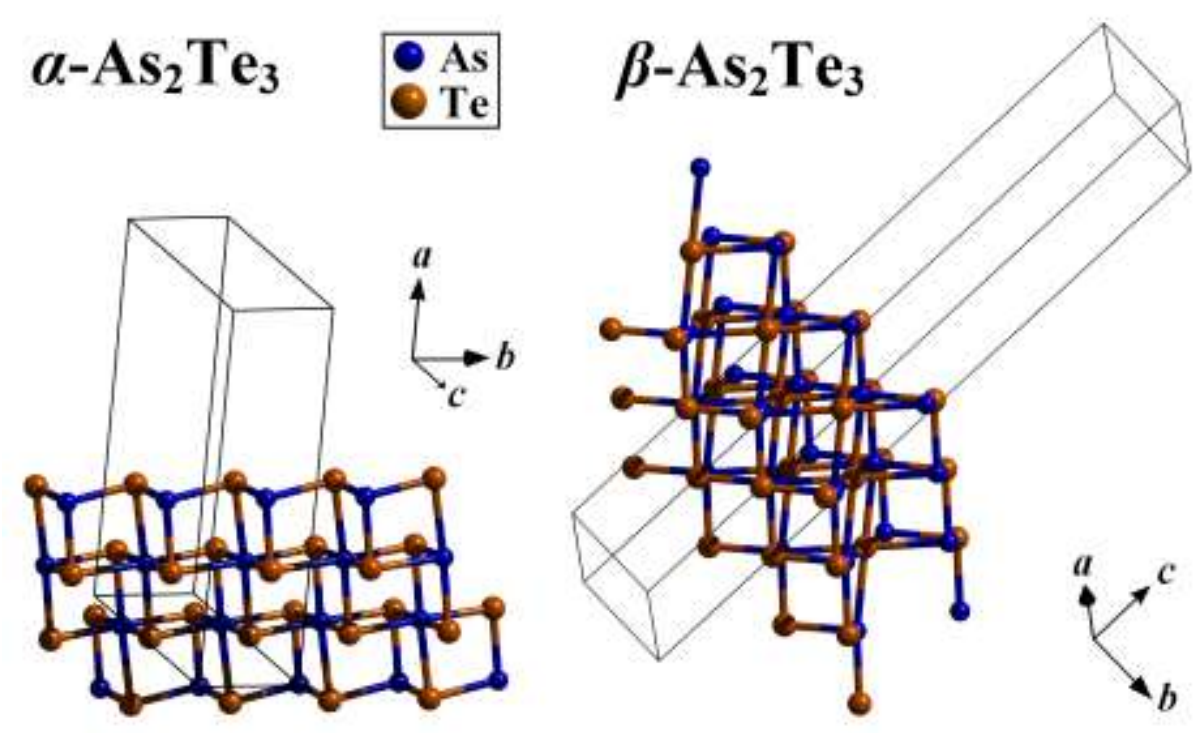

Figure S2. Schematic views of the $\left[A s_{4} T e_{6}\right]_{n}$ ribbon structure in phase $\alpha$ and the $T e(1)-A s-T e(2)-A s-T e(1)$ quintuple-layer structure in phase $\beta$ of $\mathrm{As}_{2} \mathrm{Te}_{3}$. The black bold lines denote the unit cells.

\section{Notes:}

In Figure S2, the $\left[A s_{4} \mathrm{Te}_{6}\right]_{n}$ ribbon in phase $\alpha$ and the $\mathrm{Te}(1)-A s^{-} \mathrm{Te}(2)-A s^{-} \mathrm{Te}(1)$ quintuple layer in phase $\beta$ exhibit the 4 unit cells along the $b$-axis and the $3 \times 3$ unit cells parallel the $a b$-plane, respectively. The unit cell edges of these two phases are retained, in order to view distinctly the positions of ribbon or layer in the crystal lattice. Although the crystal structure of phase $\alpha$ of $\mathrm{As}_{2} \mathrm{Te}_{3}$ is different from that of phase $\beta$, the atomic arrangement in the $\left[A s_{4} T e_{6}\right]_{n}$ ribbon in the former is similar to that in the quintuple layer in the latter. In the local ribbon or layer structures, As and Te atoms form two interpenetrating face-centered cubic (FCC) lattices, which is similar to that in the rock salt structure. Compared with the perfect rock salt structure, the distortions exist in the local structures of $\alpha$ - and $\beta$ - $\mathrm{As}_{2} \mathrm{Te}_{3}$. Although the $\left[A s_{4} T e_{6}\right]_{n}$ ribbon in phase $\alpha$ is infinite along the $b$-axis, the atoms stack only one and two layers along the $a$ - and $c$-axes, respectively. However, the $T e(1)-A s-T e(2)-A s-T e(1)$ quintuple layer in phase $\beta$ is an infinite two-dimensional structure parallel the $a b$-plane, in which the atoms stack five layers along the $c$-axis. Compared with the two-dimensional quintuple layer, the $\left[\mathrm{As}_{4} \mathrm{Te}_{6}\right]_{n}$ structure is only a one-dimensional ribbon composed of rock-salt-like As and Te atoms. 